\title{
ABOUT THE MODERNIZED GRINDING WHEEL DRESSINGDEVICE'S DIAMOND-PIN DRIVING SYSTEM BY THE NILESGEAR GRINDING MACHINES
}

\author{
Loránd MÁRTON \\ Sapientia - Hungarian University of Transylvania, Faculty of Technical and Human Sciences, Targu Mures, \\ Romania,m.lorand93@gmail.com
}

\begin{abstract}
This paper deals with criteria and conditions that must be fulfilled by a modern grinding wheel dressing system. Its main task is the correct drawing of the given profile. By this task, the diamond pin's axis must close during the motion at a constant angle with the normal of the wheel's surface. This study presents the build-up and the proposed control strategy of the dressing device. Two control methods were analysed and compared.
\end{abstract}

Keywords: Niles, grinding wheel, dressing, stepper motor, control.

\section{The operating conditions of the grinding wheel dressing device}

The modernized wheel dressing system's key parts are the high precision linear positioning tables that move the diamond pin along the profile of the grinding wheel. The main reason for the refurbishment of the classical device is the enlargement of the realizable geometries.

While performing the positioning task, the diamond tip's axis presents a double inclination defined by two angles. The first has a value between 5 and 12 degrees and is measured from the surface normal. The inclination must be in the opposite sense to the tangential velocity vector that appears with the rotation of the grinding wheel [1]. The other is a tilt [2] with a recommended value of 10-15 degrees relative to the surface normal, but in the direction of the feed motion. By this the surface quality of the cut wheel increases.

In order to keep the angular settings mentioned above, the pin's feed motion must start every dressing cycle from the same extremity of the profile. That means that a return without cutting is necessary, which requires increased machining time.

The selection of the diamond tip depends on the machining strategy to be applied to a given wheel. In the case of modernized equipment the best choice is a diamond tip with several edges.

When the edge reaches the admissible wear value it will be turned around its axis with a given angular step in order to activate the next edge [2]. In this way, the durability of the diamond tip increases several times. As a result of this, additional adjustments during the wheel dressing process becomes unnecessary.

The length of the diamond fixing holder must be as short as possible to keep the amplitude of vibrations on a possible minimal level. This will avoid cracking of the diamond tip during the machining process..

\section{Structural examination}

The first result of the conceptual design consists of the holder shown in Figure 1. This will be mounted on the Niles grinding machine body in the place of the old wheel dressing system.

First of all, in order to avoid useless correctional computing or manufacturing errors that appear as profile distortion, the diamond tip must move in a vertical plane that passes through the grinding wheel's axis. This condition is fulfilled by implementing a fine positioning system mounted on that controlled slider, which executes the vertical 


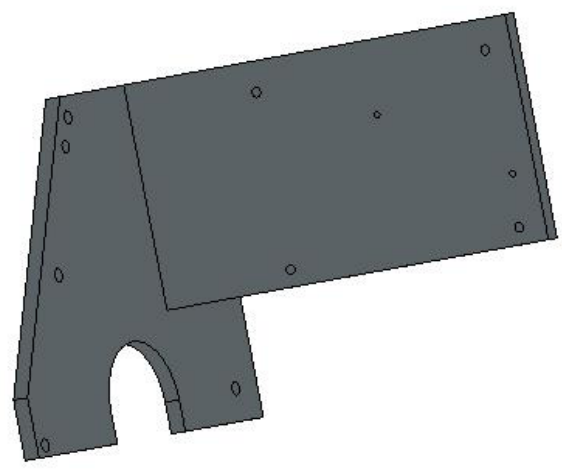

Figure 1. The holding stand

motion (perpendicular to the grinding wheel's axis). This movement is perpendicular to the vertical plane defined before. Thus, the position of the diamond tip can be corrected after each replacement.

Geometrical errors of the holder - these are systematic errors - should be taken into consideration when writing the control program of the slider motions.

Such errors are the perpendicularity deviation of the holder plate from the support plate and the parallelism error of the mounting. The perpendicularity deviation of the sliders from each other are neglected since it is a fine mechanical device manufactured by the Parker-Hannifin company (who guarantee the maximal deviation under one micron over a length of $100 \mathrm{~mm}$ ).

Systematic errors are unavoidable in the assembling process of the control equipment. These errors will be identified through a fine measuring process and compensated for by introducing the necessary corrections in the control program. As a consequence of the facts described above, the real profile of the grinding wheel results in dependence on the limits of the resolution and the accuracy of the system. It should be noted, that the fitting precision and the geometrical position accuracy must be considered as statistical variables until they are obtained through measurement. The program uses as input values the mean values of the statistic variables associated with the control dimensions of the equipment.

\section{An overview of the possible solutions}

The diamond pin' support is a subsystem of the dressing equipment that must be linked to the controller of the stepper motor in order to correlate the position of the diamond pin axis with the linear positioning units.
The first solution that allows the perpendicularity of the diamond pin on the wheel profile is a pneumatic, PLC controlled system. This fulfills the imposed motion task with the help of two cylinders and a rack-gear mechanism.

The second solution is an improved one that avoids the deficiencies and inflexibility of the pneumatic system. The system becomes more versatile by implementing a stepper motor connected to a gear drive that continuously moves the diamond pin's axis in the required position.

\subsection{The pneumatic rotational unit}

The task diagram of the pneumatic positioning system is shown in Figure 2. The two pneumatic cylinders allow three distinct positions of the diamond pin (Figure 3.). In the extreme positions, the axis of the diamond tip closes an angle of 70 degrees with the vertical direction, while in the central position it is perpendicular to the axis of the grinding wheel. By this last position the correction of the wheel topland is performed.

All wheel profiles that differ from the standard $20^{\circ}$ pressure angle defined rectilinear shape can be drawn by applying a continuous correctional motion. But the diamond tip axis keeps the $70^{\circ}$ setting angle shown in Figure 3.

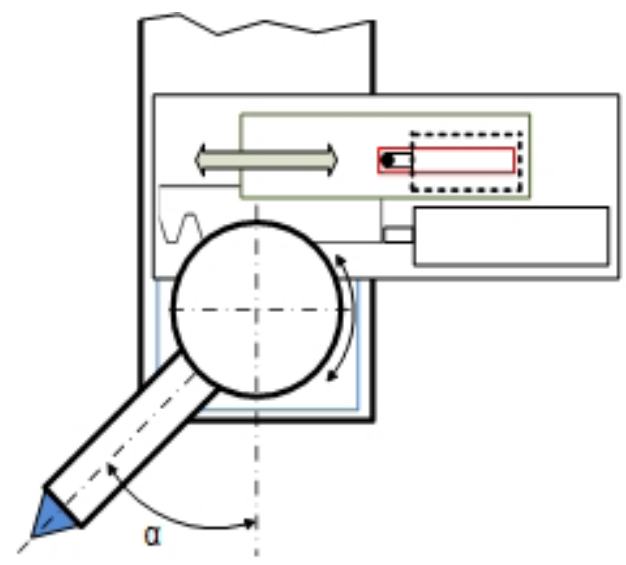

Figure 2. An outline of the pneumatic implementation of the tool holder position change system
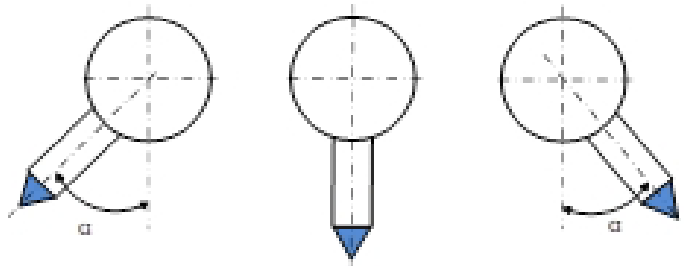

Figure 3. The positions occupied by the diamond tip 
If no supplementary correction is applied, the diamond tip executes a linear motion and draws the standard $20^{\circ}$ pressure angle shaped profile.

The diamond tip axis position changes due to the simultaneous action of the pneumatic cylinders. Their strokes are of different lengths with a ratio of 1:2.

When the piston that executes the shorter stroke reaches the lower end position, the other piston starts moving. Here a collision danger could appear. This is avoided by implementing a sliding bolt on the unit that can move in a nut.

\subsubsection{The disadvantage of the proposed wor- king cycle and sharpening strategy}

The working cycle of the pneumatic system presented above may consist of the following steps:

- approaching the left side, correction;

- diamond position change, approaching the topland;

- diamond shaft position change; approaching the right side, correction;

- lifting out, reversing and lowering on the right side.

Due to the design of the diamond holder, the feed can be realized in only one direction, since the diamond holder's axis closes a constant angle with the perpendicular plane.

The inflexibility becomes visible when we try to create a curved grinding wheel profile that is mainly different from a standard profile $[3,4,5]$. Because of the inflexibility of the system, it is unable to follow a curved line, thus the initial setting conditions cannot be kept. This compromises the integrity of the diamond pin and results in a bad quality of the machined surface.

\subsection{The stepper motor driven position changing system}

The limits of the solution involving pneumatic cylinders make this unable to meet the compulsory technological conditions. Thus, development of a new solution is necessary. The improved system uses a stepper motor that drives a gear pair which rotates the axis of the diamond in real time in the correct position, imposed by the direction of the surface normal vector.

When selecting the stepper motor, it is essential that it is able to operate at high speeds with high precision. The ideal choice for this purpose is a hybrid bipolar stepper motor.

The diamond tip control unit must be able to communicate with the linear motion controller.
In addition, it must function also in a micro stepper mode, in order to reduce the stepping angle to increase the profile accuracy.

\subsubsection{Structural examination}

The diamond tool positioning control acts on the stepper motor. A gear drive will continuously correct the diamond tip axis position.

A kinematic sketch of the device is shown in Figure 4. Due to the rotation of the grinding wheel, the main load on the diamond tip holder consists of an axial compression. Thus axial radial roller bearings were built in. The device is fixed to the vertically moving linear positioning table with pins and screws.

During the cutting process a considerable heat occurs. As a consequence the diamond tip must be cooled continuously. The coolant will be sprayed directly on it.

The spraying nozzle is also fixed to the vertically moving linear positioning table.

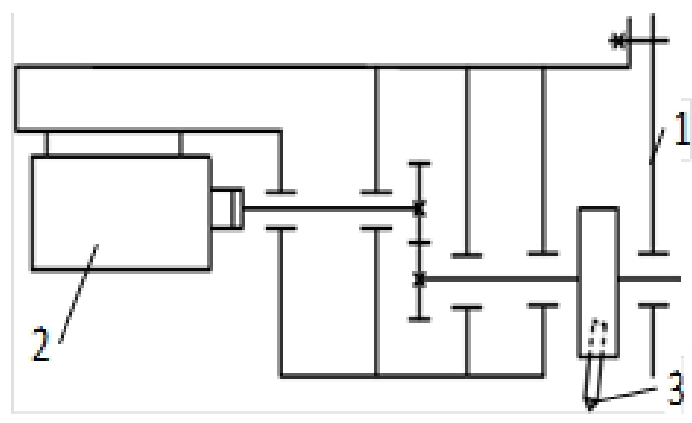

Figure 4. Tool position changer, (1) positioning table, (2) stepper motor, (3) diamond tip and clamp

\subsubsection{The control cycle}

The advantage of the second solution consists of a much more efficient control cycle. While the positioning of the diamond tip no longer requires the return to the base-line, it can continue the dressing procedure from any side. The cycle structure is illustrated in Figure 5. and consists of the following steps:

- approaching the left side, correction;

- topland correction;

- right side correction;

- lifting, waiting, lowering, and correcting in reversed order;

The technological purpose will be achieved by the implementation of this solution. The system gains more flexibility. The profile of the grinding wheel can be easily modified in order to fit any modified rack profile. 


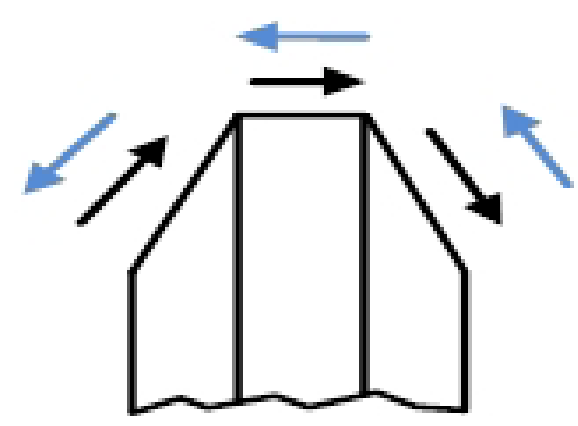

Figure 5. The control cycle

\section{Conclusions}

As a result of the first concept, a pneumatic system was designed to position the diamond tip. After a deeper analysis of the problem, it is seen that this is not adequate until the diamond pin axis direction can no longer be adapted to the conditions imposed by any profile. Its position angle remains constant along the dressing cycle.

The pneumatic solution is relatively simple, but its possibilities are limited. In opposition to this, the stepper motor is the relevant solution, although its control is complicated. As a compen- sation, a much greater versatility is offered while meshing arbitrary curved profiles.

The next task consists of designing a mechanism that allows the easy fixing of the diamond tip and the accurate rotation around its axis. This is necessary because the cutting process produces a large amount of dust.

\section{References}

[1] Tawakoli T., Rasifard A.: Dressing of Grinding Wheels. In: Machining with Abrasives (eds. Jackson M. J., Davim J. P.) Springer, 2011, 181-244.

[2] Köves E., Móser M., Almásy P.: Köszörülés és finommegmunkálás. Müszaki Könyvkiadó, Budapest, 1973.

[3] Dudás, I.: The theory and practice of worm gear drives. Butterworth-Heinemann, 2005.

[4] Dudás,I., Bodzás, S.: Measuring technique and mathematical analysis of conical worms. The International Journal of Advanced Manufacturing Technology 66/9-12. (2013) 2075-2085. https://doi.org/10.1007/s00170-012-4483-7

[5] Dudás, I., Balajti, Zs.: A New Description Method for the Bearing Pattern of the Spiroid Driving. In: ITC 2005 4th International Tools Conference, Zlin24-25. 05. 2005., University of Zlín, CZ, 8-15. 